\title{
Real world experience in low-dose ipilimumab in combination with PD-1 blockade in advanced melanoma patients
}

\author{
Michael Constantin Kirchberger ${ }^{1}$, Alvaro Moreira ${ }^{1}$, Michael Erdmann ${ }^{1}$, Gerold \\ Schuler $^{1}$ and Lucie Heinzerling ${ }^{1}$ \\ ${ }^{1}$ Department of Dermatology, University Hospital Erlangen, Friedrich-Alexander-University Erlangen-Nürnberg (FAU), \\ Ulmenweg 18, 91054 Erlangen, Germany \\ Correspondence to: Lucie Heinzerling, email: Lucie.Heinzerling@uk-erlangen.de \\ Keywords: ipilimumab; pembrolizumab; nivolumab; low-dose; melanoma \\ Received: February 04, $2018 \quad$ Accepted: May 30, $2018 \quad$ Published: June 22, 2018 \\ Copyright: Kirchberger et al. This is an open-access article distributed under the terms of the Creative Commons Attribution License \\ 3.0 (CC BY 3.0), which permits unrestricted use, distribution, and reproduction in any medium, provided the original author and \\ source are credited.
}

\section{ABSTRACT}

Dual immune-checkpoint blockade with the anti-PD-1 antibody nivolumab (1 $\mathrm{mg} / \mathrm{kg}$ ) and standard-dose ipilimumab $(3 \mathrm{mg} / \mathrm{kg})$ is the mainstay of immunotherapy in advanced melanoma and it is approved since 2016. However, severe side effects (grade $3 / 4$ ) occur in up to $60 \%$ of the patients. Recently, clinical trials have shown similar anti-tumor activity with a more favorable toxicity profile in patients treated with low-dose ipilimumab $(1 \mathrm{mg} / \mathrm{kg})$ and standard-dose pembrolizumab $(2 \mathrm{mg} /$ $\mathrm{kg})$. In this study we report on the real-world experience of this dosing regime in advanced melanoma patients not eligible for clinical trials. A total of 33 patients with metastatic melanoma ( 24 with cutaneous and 9 with uveal melanoma) were assessed, retrospectively. Brain metastases were present in $33 \%$ of the patients and lactate dehydrogenase was elevated in $70 \%$. Overall response rates were $38 \%$ and $0 \%$ in cutaneous melanoma and uveal melanoma respectively. Median overall survival was not reached in cutaneous melanoma and was 18 months in uveal melanoma. In $18 \%$ of the patients at least one treatment-related severe adverse event was observed. Our observation that the combination of standard dose pembrolizumab and low-dose ipilimumab has a favorable toxicity profile yet anti-tumor activity comparable to the approved standard-dose combination regime in advanced patients not suitable for enrollment in clinical trials is encouraging.

\section{INTRODUCTION}

In recent years, monoclonal antibodies blocking the inhibitory programmed cell death 1 pathway (PD-1/PDL1) and the cytotoxic $\mathrm{T}$ lymphocyte associated protein 4 (CTLA-4) have significantly impacted the treatment of advanced melanoma. The objective response rates for the anti-CTLA-4 antibody ipilimumab, the antiPD-1 antibodies nivolumab and pembrolizumab, and the combined blockade of CTLA-4 and PD-1 are 6\%-19\%, $21 \%-44 \%$, and $53 \%-61 \%$, respectively [1-7]. In patients with brain metastases intracranial and overall response rate was $42 \%$ and $55 \%[8,9]$. However, the combination of CTLA-4 and PD-1 blockade also induces considerable toxicity with the highest frequency of grade $3 / 4$ adverse events (AEs) (53\%-59\%) compared to anti-PD-1 (21\%) or -CTLA-4 monotherapy $(28 \%)[4,10,11]$.

Several clinical trials are modifying treatment protocols to optimize the cost-benefit ratio of response rate, survival and toxicity of checkpoint inhibitors. In metastatic renal cell carcinoma efficacy as assessed by ORR and progression-free survival (PFS) did not differ between ipilimumab $3 \mathrm{mg} / \mathrm{kg}$ + nivolumab $1 \mathrm{mg} / \mathrm{kg}$ and ipilimumab $1 \mathrm{mg} / \mathrm{kg}$ + nivolumab $3 \mathrm{mg} / \mathrm{kg}$, however, the low-dose ipilimumab group showed a better safety profile [12]. In melanoma the low-dose ipilimumab (1 $\mathrm{mg} / \mathrm{kg}$ ) protocol was investigated in the KEYNOTE-029 (NCT02089685) and CheckMate 511 (NCT02714218) trials. Long et al. published results (KEYNOTE-029) of 153 patients showing an ORR of $61 \%$ and a disease control 
rate (DCR) of $79 \%$ with $45 \%$ grade $3 / 4$ treatment-related [13]. Reducing the toxicity by the sequential administration of Ipilimumab (3 $\mathrm{mg} / \mathrm{kg}$, four doses), followed by nivolumab (3 $\mathrm{mg} / \mathrm{kg})$ versus vice versa has also been investigated (Checkmate 064 trial) [14]. Notably, the rate of grade 3/4 AEs between both cohorts was also high with $63 \%$ in the nivolumab followed by ipilimumab group and $50 \%$ in the reverse sequence. Reducing the number of ipilimumab infusions was investigated in a cohort of 40 patients with short-term ipilimumab (2 cycles) followed by PD-1 blockade and induced treatment-related grade $3 / 4$ AEs in only $38 \%$ of patients with an ORR of $55 \%$ [15]. Thus, sequencing and dosing of anti-CTLA-4 and anti-PD-1 plays a significant role regarding efficacy and toxicity. However, the best regime is still to be determined.

This study analyzes the clinical course of 33 patients with advanced metastatic melanoma who received the combination of low-dose ipilimumab (1 $\mathrm{mg} / \mathrm{kg})$ and standard-dose anti-PD-1 antibodies $(2 \mathrm{mg} / \mathrm{kg})$ in a realworld setting. We report clinical outcomes with respect to response, survival and tolerability.

\section{RESULTS}

A total of 33 patients were included in this study (Figure 1), 23 patients with cutaneous melanoma, 9 patients with uveal melanoma and 1 patient with melanoma of unknown primary (MUP; Table 1 ). The patient with MUP harbored a v-Raf murine sarcoma viral oncogene homolog (BRAF) V600E mutation and was assigned to the group of cutaneous melanomas. Median follow-up was 12 months with a cutoff date as of September 2017.

In $17 \%(N=4)$ of the patients with cutaneous melanoma, prior treatment was recorded and consisted of targeted therapy with BRAF and MEK inhibitors (33\% of BRAF mutant patients; $N=4$ ). One patient had radiochemotherapy prior to targeted therapy. Treatment stop of prior treatment was due to progressive disease in all of the cases.

In $89 \%(N=8)$ of the patients with uveal melanoma, hepatic metastases were present and prior treatment with either selective internal radiotherapy $(N=4)$, radiofrequency ablation $(N=1)$ or microwave ablation $(N=1)$ was performed. In two cases, these therapeutic modalities were omitted due to the extent of metastases.

First line immunotherapy was combined pembrolizumab (2 $\mathrm{mg} / \mathrm{kg}$ ) and low-dose ipilimumab (1 $\mathrm{mg} / \mathrm{kg}$ ) for 4 infusions every three weeks in all patients. In cutaneous melanoma $(N=24)$ ORR was $38 \%(N=9)$ and DCR $67 \%(N=16$; Table 2$)$. Patients with uveal melanoma had an ORR of $0 \%(N=0)$ and

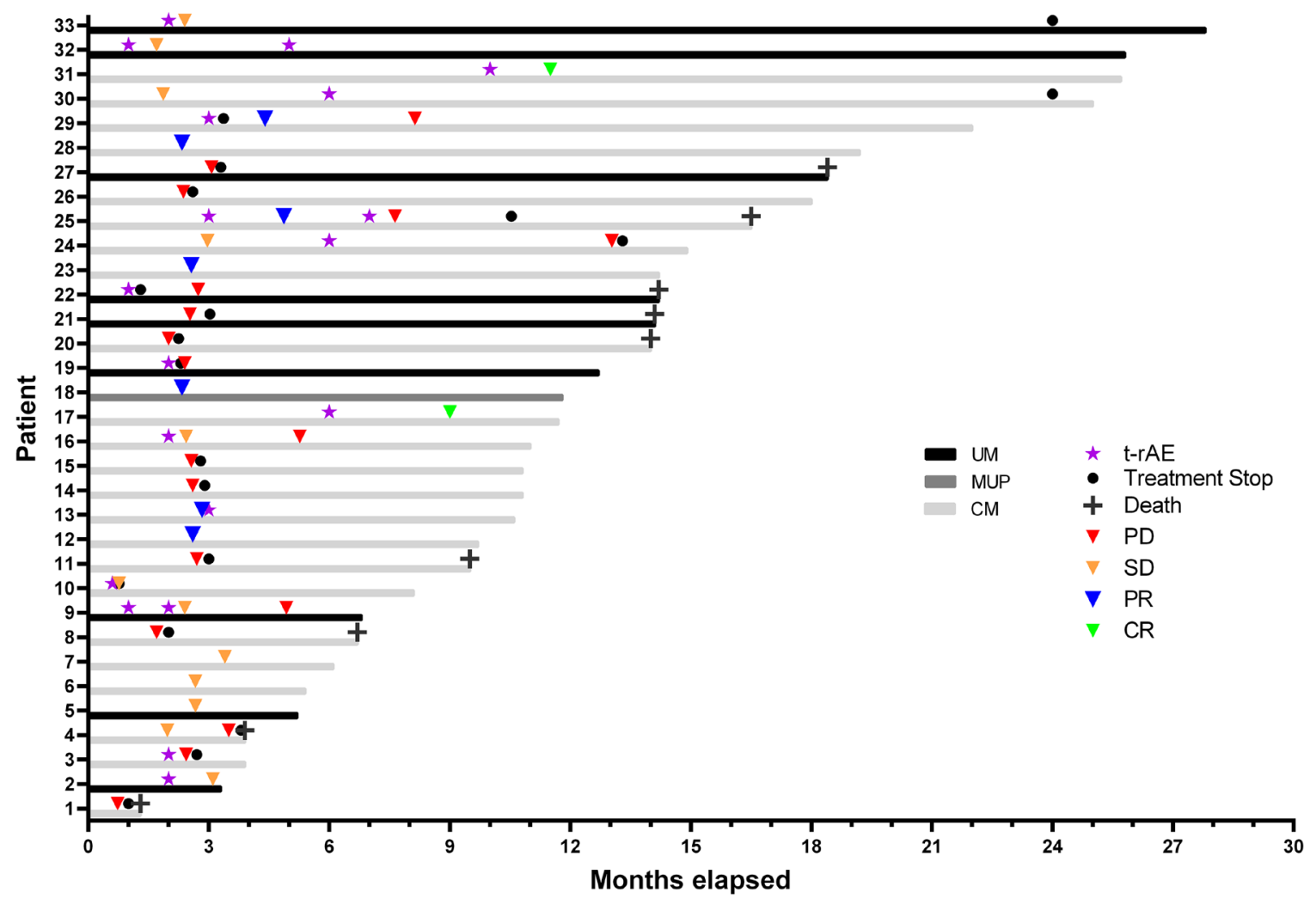

Figure 1: Swimmers plot of melanoma patients treated with low-dose ipilimumab and standard-dose pembrolizumab. $\mathrm{UM}=$ uveal melanoma $(\mathrm{N}=9), \mathrm{MUP}=$ melanoma of unknown primary $(\mathrm{N}=1), \mathrm{CM}=$ cutaneous melanoma $(\mathrm{N}=23), \mathrm{t}$-rAE $=$ treatmentrelated adverse event, $\mathrm{PD}=$ progressive disease, $\mathrm{SD}=$ stable disease, $\mathrm{PR}=$ partial response, $\mathrm{CR}=$ complete response. 
Table 1: Baseline characteristics of the patients

\begin{tabular}{|c|c|c|}
\hline Characteristic & Cutaneous Melanoma & Uveal Melanoma \\
\hline \multicolumn{3}{|l|}{ Age at stage IV (years) } \\
\hline Median (range) & $57(32-87)$ & $66(54-75)$ \\
\hline \multicolumn{3}{|l|}{ Sex, N (\%) } \\
\hline Female & $6(24)$ & $3(33)$ \\
\hline Male & $18(75)$ & $6(67)$ \\
\hline \multicolumn{3}{|l|}{ ECOG performance status } \\
\hline 0 & $15(63)$ & $4(45)$ \\
\hline 1 & $7(29)$ & $3(33)$ \\
\hline 2 & $2(8)$ & $2(22)$ \\
\hline \multicolumn{3}{|l|}{ Metastatic site, $\mathbf{N}(\%)$} \\
\hline Brain & $10(42)$ & $1(11)$ \\
\hline Liver & $8(33)$ & $8(88)$ \\
\hline Lung & $11(46)$ & $3(33)$ \\
\hline Nodal/cutaneous & $18(75)$ & $3(9)$ \\
\hline Bone & $6(25)$ & $0(0)$ \\
\hline Other & $6(25)$ & $1(11)$ \\
\hline \multicolumn{3}{|l|}{ Mutation status, N (\%) } \\
\hline NRAS/BRAF wildtype & $10(42)$ & NA \\
\hline BRAFV600 & $12(50)$ & NA \\
\hline NRAS & $2(8)$ & NA \\
\hline \multicolumn{3}{|l|}{ Prior treatment, N (\%) } \\
\hline None & $20(83)$ & $1(11)$ \\
\hline BRAF- and MEK-Inhibitors & $4(17)$ & $0(0)$ \\
\hline Chemotherapy & $1(4)$ & $0(0)$ \\
\hline Liver-specific therapies & $0(0)$ & $8(89)$ \\
\hline \multicolumn{3}{|l|}{ Lactate dehydrogenase } \\
\hline Median (range) & $339(160-2353)$ & $278(216-407)$ \\
\hline$\leq \mathrm{ULN}(\mathrm{N},(\%))$ & $6(25)$ & $4(44)$ \\
\hline$>\mathrm{ULN}$ & $18(75)$ & $5(56)$ \\
\hline \multicolumn{3}{|l|}{ S100B } \\
\hline Median (range) & $0.24(0.03-22.65)$ & 0.06 \\
\hline$\leq \mathrm{ULN}(\mathrm{N},(\%))$ & $6(25)$ & $8(89)$ \\
\hline$>\mathrm{ULN}$ & $18(75)$ & $1(11)$ \\
\hline
\end{tabular}




\begin{tabular}{lcc}
\hline Characteristic & Cutaneous Melanoma & Uveal Melanoma \\
\hline MIA & $23.1(2.9-161.2)$ & $10.1(4.3-30.4)$ \\
Median (range) & $7(33)$ & $4(50)$ \\
$\leq$ ULN (N, (\%)) & $14(67)$ & $4(50)$ \\
$>$ ULN & & $1.5(1.1-11.9)$ \\
CRP & $5.8(3.3-105.3)$ & $2(67)$ \\
Median (range) & $7(41)$ & $1(33)$ \\
$\leq$ ULN (N, (\%)) & $10(59)$ & $1(0.1-7.0)$ \\
$>$ ULN & & $2(0.1-5.0)$ \\
REC (\%) & & \\
Median (range) & &
\end{tabular}

Abbreviations: $\mathrm{ECOG}=$ Eastern Cooperative Oncology Group, $\mathrm{BRAF}=\mathrm{v}-\mathrm{Raf}$ murine sarcoma viral oncogene homolog, NRAS = neuroblastoma rat sarcoma viral oncogene homolog, S100B $=$ S100 calcium-binding protein B, MIA = melanoma inhibitory activity, $\mathrm{CRP}=\mathrm{c}$-reactive protein, $\mathrm{REC}=$ relative eosinophil counts, $\mathrm{ULN}=$ upper limit of normal.

Table 2: Treatment response to combined immunotherapy with ipilimumab and pembrolizumab in cutaneous and uveal melanoma

\begin{tabular}{lcc}
\hline Best Response, $\mathbf{N}(\%)$ & Cutaneous Melanoma $(\mathbf{N}=\mathbf{2 4})$ & Uveal Melanoma (N = 9) \\
\hline PD & $8(33)$ & $4(44)$ \\
SD & $7(29)$ & $5(56)$ \\
PR & $7(29)$ & $0(0)$ \\
CR & $2(8)$ & $0(0)$ \\
\hline
\end{tabular}

Abbreviations: $\mathrm{PD}=$ progressive disease, $\mathrm{SD}=$ stable disease, $\mathrm{PR}=$ partial response, $\mathrm{CR}=$ complete response .

a DCR of $56 \%(N=5)$. Median time to best response and treatment stop was 3 months. Monotherapy with pembrolizumab $(2 \mathrm{mg} / \mathrm{kg})$ was continued after combined immunotherapy in $46 \%(N=13)$ of the patients with cutaneous melanoma and $44 \%(N=4)$ of the patients with uveal melanoma. The median overall survival (OS) from initiation of ipilimumab and pembrolizumab was 18.4 months in the uveal melanoma group and was not reached for cutaneous melanoma (Figure 2; $P=$ $0.005)$. The most common reasons for discontinuation of treatment were disease progression $(42 \% ; N=14)$ and adverse events (AEs; $18 \% ; N=6$ ). Two patients $(6 \%)$ discontinued therapy after 24 months and achieved SD during the treatment period.

In $61 \%(N=20)$ of the patients at least one treatment-related $\mathrm{AE}$ was recorded, including $18 \%(N$ $=6$ ) who had at least of $\mathrm{AE}$ of grade 3 or 4 severity. No treatment-related deaths occurred. Most common treatment-related AEs comprised colitis, diarrhea, thyroiditis and vitiligo with a median time of onset after 10 weeks (Figure 3). In 10 patients, treatment-related AEs occurred before response (SD, PR or CR) to therapy with a median time of onset of 4 weeks after the AE.

\section{DISCUSSION}

In this study we show that combined standard-dose pembrolizumab (2 $\mathrm{mg} / \mathrm{kg}$ ) with low-dose ipilimumab (1 $\mathrm{mg} / \mathrm{kg}$ ) has a strikingly better toxicity profile yet similar anti-tumor activity as standard combination therapy. ORR was $38 \%$ in this patient cohort with unfavorable prognostic markers (33\% brain metastases, $70 \%$ elevated LDH) compared to $58 \%$ and grade $3 / 4$ side effects occurred in only $18 \%$ compared to $59 \%$ [11]. However, in this real-world setting, registration of AEs is somewhat less than in clinical trials. Reducing severe treatmentrelated AEs remains a main concern and multiple studies are evaluating whether or not reduction of the dose or frequency of administration can improve tolerability at similar efficacy $[13,16-18]$. Interestingly, our rate of grade $3 / 4$ side effects with $18 \%$ was even lower than the $45 \%$ in the Keynote-029 trial [13]. However, this difference could be attributed to the small cohort and the less rigorous surveillance of AEs in a real-world setting. Also differences between subgroups might have been missed due to the limited cohort size. 


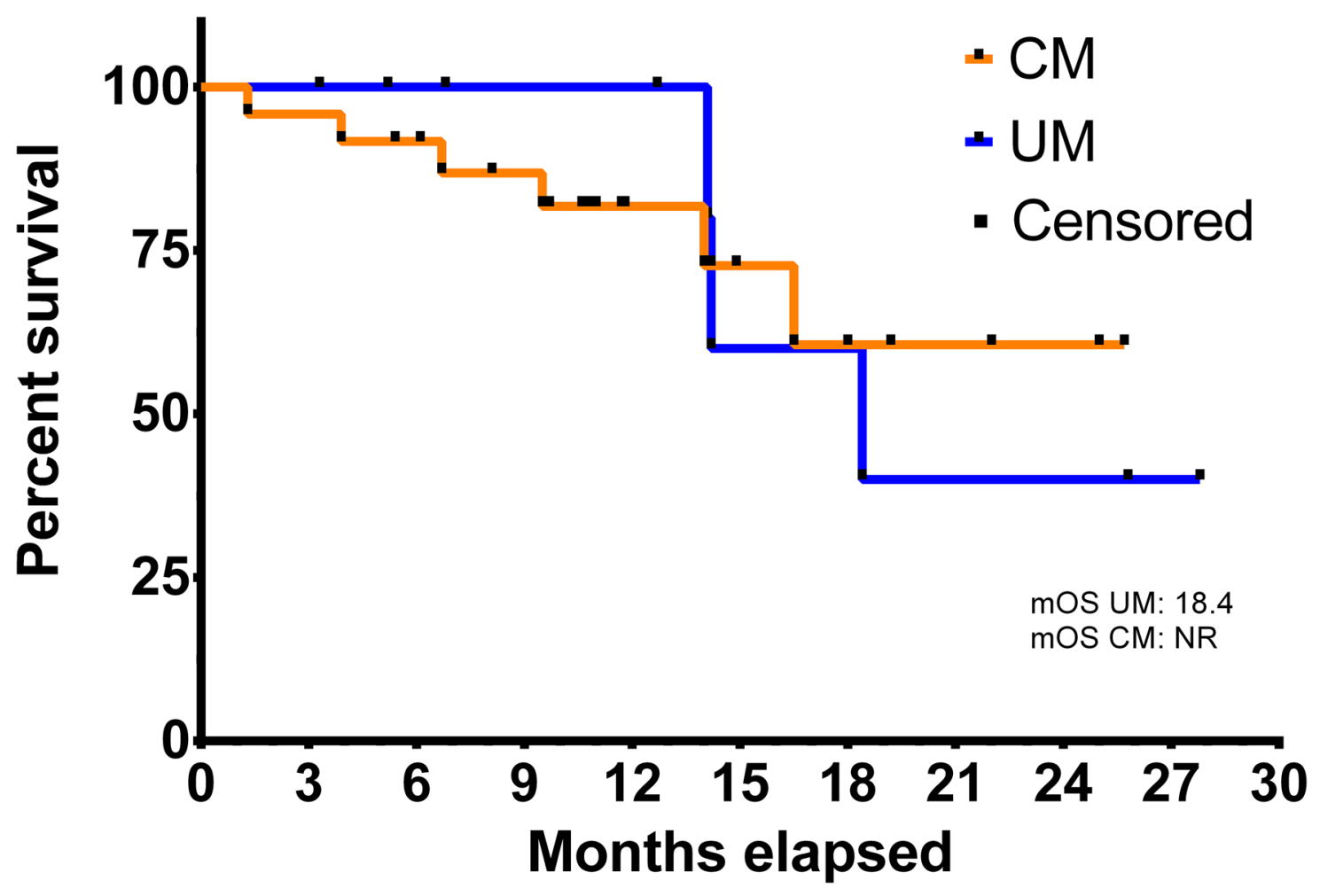

Figure 2: Overall survival from initiation of checkpoint inhibitor therapy in uveal $(\mathrm{UM}, \mathrm{N}=9)$ and cutaneous melanoma (CM, $\mathbf{N}=\mathbf{2 4})$. $\mathrm{mOS}=$ median overall survival, $\mathrm{NR}=$ not reached.

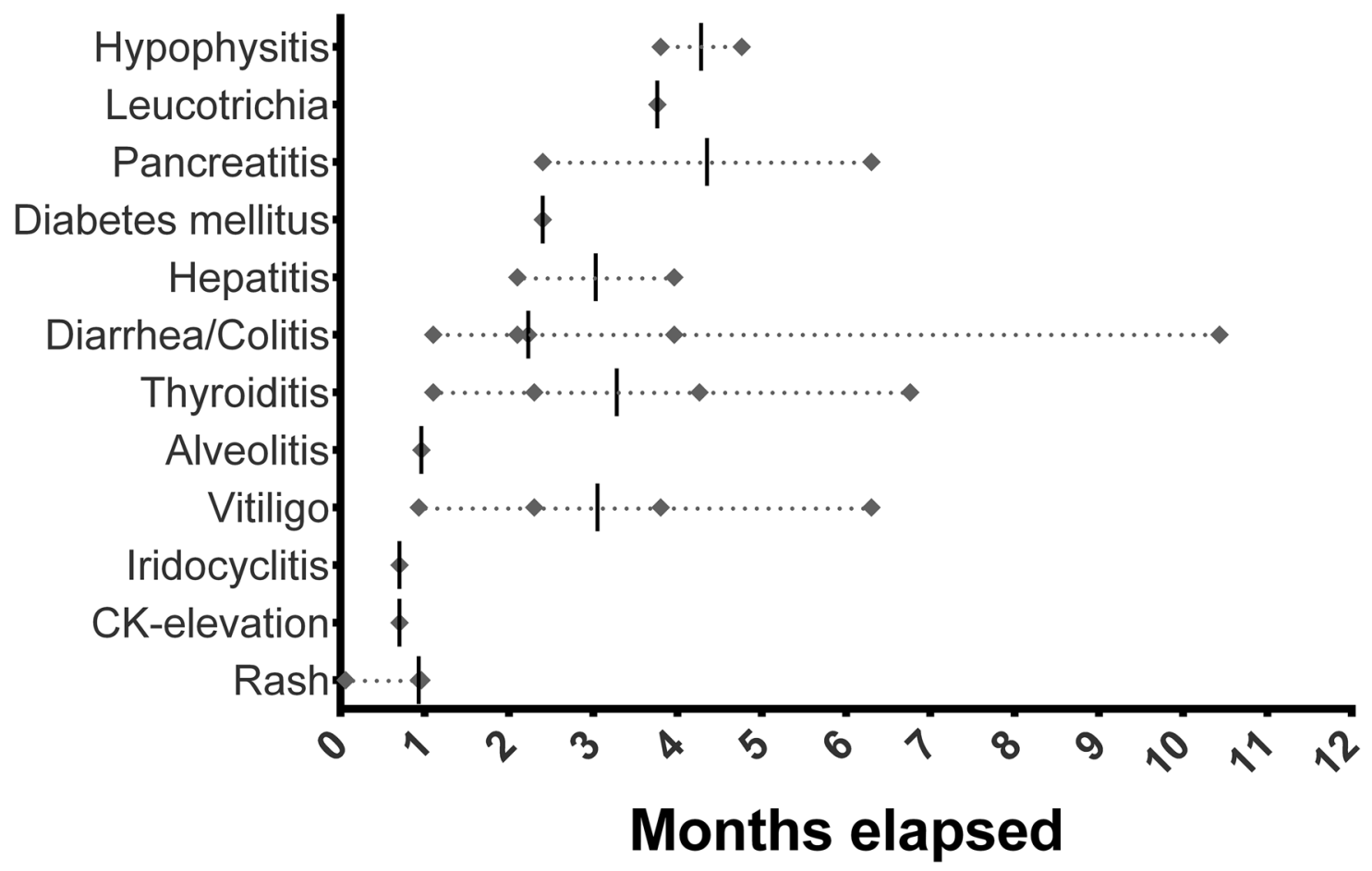

Figure 3: Temporal occurrence of treatment-related adverse events (Diamond) in months. Bar $=$ median, $\mathrm{CK}=\mathrm{creatine}$ kinase. 
In terms of disease control, our cohort showed a DCR of $67 \%$ in cutaneous melanoma compared to $79 \%$ in the Keynote-029 trial [13]. Baseline characteristics may account for that difference since patients in our cohort were not suitable for participation in clinical trials. In cutaneous and uveal melanoma elevated LDH was observed in $75 \%$ and $56 \%$, respectively. Also brain metastasis was present in $44 \%$ of the cutaneous melanoma and in $11 \%$ of the uveal melanoma.

Interestingly, in 10 patients treatment-related AEs were observed before, and in 1 patient shortly after positive response to immunotherapy, compared to 5 patients who suffered PD after treatment-related AEs. Vitiligo as treatment-related AE is typically observed in melanoma patients treated with anti-PD-1 antibodies [19] and indicated positive response to therapy in 4 of our patients (Figure 1, Patient 2, 9, 25, 30). However, the predictive use of treatment-related AEs to therapy response has to be further investigated.

\section{MATERIALS AND METHODS}

This retrospective analysis included checkpointblockade naïve advanced cutaneous and uveal melanoma patients treated with combined immune checkpoint inhibitors ipilimumab ( $1 \mathrm{mg} / \mathrm{kg}, \mathrm{q} 3 \mathrm{w}$ in combination) and pembrolizumab (2 $\mathrm{mg} / \mathrm{kg}$, subsequently q3w) at the skin cancer center Erlangen between January 2016 and September 2017. Patients were screened using pharmacy records and clinical data and treatment outcomes were extracted from pre-existing patient records in the eligible cases.

Clinical data taken at baseline before initiation of the therapy comprised demographics with Eastern Cooperative Oncology Group (ECOG) performance status, mutation status, metastatic sites, and prior systemic antineoplastic therapies. In addition, laboratory parameters such as lactate dehydrogenase (LDH), C-reactive protein (CRP), eosinophils (REC), S100B and melanoma inhibitory activity (MIA) were gathered and analyzed.

Tumor response was assessed every 3 months by CT- and MRI-scans by a radiologist according to the Response Evaluation Criteria in Solid Rumors (RECIST) version 1.1. to determine DCR (CR rate plus $\mathrm{PR}$ rate plus rate of $\mathrm{SD}$ ) and $\mathrm{ORR}$ (CR rate plus $\mathrm{PR}$ rate). However, if progressive disease was suspected clinically or due to increasing tumor markers, imaging was performed immediately. Overall survival and PFS were calculated as time from the initiation of the therapy until melanoma-specific or treatment-related death and disease progression, respectively. They were computed with timeto-event analyses with death and progression considered as events. If no such event occurred or if patients were lost to follow-up, the date of the last documented contact was registered and used as censored observation. The survival and progression probabilities were calculated with the Kaplan-Meier method for censored failure time data assuming proportional hazards.

Toxicity was graded based on the patient records and clinical outcomes according to the Common Terminology Criteria for Adverse Events (CTCAE) v4.03 published by the National Institutes of Health in 2010. In addition, treatment-related deaths and treatment discontinuation due to severe AE were specifically collected. Patients completed a questionnaire to screen for AEs at the time of each infusion. A physician controlled the questionnaire and performed a physical examination.

This study was approved by the institutional review board of the medical faculty of the University Erlangen and was conducted in accordance with the principles of the Helsinki Declaration of 1975.

\section{CONFLICTS OF INTEREST}

Clinical trial support to institution: BMS, MSD, Roche, Amgen and Novartis.

MCK: None; AM: None; ME: None; GS: None; LH: Consultancy fees, advisory board, speakers fees, travel grants: BMS, MSD, Roche, Amgen, Curevac and Novartis.

\section{REFERENCES}

1. Weber JS, D'Angelo SP, Minor D, Hodi FS, Gutzmer R, Neyns B, Hoeller C, Khushalani NI, Miller WH, Lao CD, Linette GP, Thomas L, Lorigan P, et al. Nivolumab versus chemotherapy in patients with advanced melanoma who progressed after anti-CTLA-4 treatment (CheckMate 037): a randomised, controlled, open-label, phase 3 trial. Lancet Oncol. 2015; 16:375-84. https://doi.org/10.1016/ S1470-2045(15)70076-8.

2. Hodi FS, O'Day SJ, McDermott DF, Weber RW, Sosman JA, Haanen JB, Gonzalez R, Robert C, Schadendorf D, Hassel JC, Akerley W, van den Eertwegh AJ, Lutzky J, et al. Improved survival with ipilimumab in patients with metastatic melanoma. N Engl J Med. 2010; 363:711-23. https://doi.org/10.1056/NEJMoa1003466.

3. Robert C, Long GV, Brady B, Dutriaux C, Maio M, Mortier L, Hassel JC, Rutkowski P, McNeil C, Kalinka-Warzocha E, Savage KJ, Hernberg MM, Lebbé C, et al. Nivolumab in previously untreated melanoma without BRAF mutation. N Engl J Med. 2014; 372:320-30. https://doi.org/10.1056/ NEJMoa1412082.

4. Robert C, Schachter J, Long GV, Arance A, Grob JJ, Mortier L, Daud A, Carlino MS, McNeil C, Lotem M, Larkin J, Lorigan P, Neyns B, et al; KEYNOTE-006 investigators. Pembrolizumab versus Ipilimumab in Advanced Melanoma. N Engl J Med. 2015; 372:2521-32. https://doi.org/10.1056/ NEJMoa1503093.

5. Larkin J, Chiarion-Sileni V, Gonzalez R, Grob JJ, Cowey CL, Lao CD, Schadendorf D, Dummer R, Smylie M, Rutkowski P, Ferrucci PF, Hill A, Wagstaff J, et al. 
Combined Nivolumab and Ipilimumab or Monotherapy in Untreated Melanoma. N Engl J Med. 2015; 373:23-34. https://doi.org/10.1056/NEJMoa1504030.

6. Robert C, Thomas L, Bondarenko I, O'Day S, Weber J, Garbe C, Lebbe C, Baurain JF, Testori A, Grob JJ, Davidson N, Richards J, Maio M, et al. Ipilimumab plus dacarbazine for previously untreated metastatic melanoma. N Engl J Med. 2011; 364:2517-26. https://doi.org/10.1056/ NEJMoa1104621.

7. Ribas A, Puzanov I, Dummer R, Schadendorf D, Hamid O, Robert C, Hodi FS, Schachter J, Pavlick AC, Lewis KD, Cranmer LD, Blank CU, O’Day SJ, et al. Pembrolizumab versus investigator-choice chemotherapy for ipilimumabrefractory melanoma (KEYNOTE-002): a randomised, controlled, phase 2 trial. Lancet Oncol. 2015; 16:908-18. https://doi.org/10.1016/S1470-2045(15)00083-2.

8. Long GV, Atkinson V, Menzies AM, Lo S, Guminski AD, Brown MP, Gonzalez MM, Diamante K, Sandhu SK, Scolyer RA, Emmett L, McArthur GA. A randomized phase II study of nivolumab or nivolumab combined with ipilimumab in patients (pts) with melanoma brain metastases (mets): The Anti-PD1 Brain Collaboration (ABC). (Presented at ASCO 2017). Journal of Clinical Oncology. 2017; 35:9508-9508.

9. Tawbi HAH, Forsyth PAJ, Algazi AP, Hamid O, Hodi FS, Moschos SJ, Khushalani NI, Gonzalez R, Lao CD, Postow MA, Atkins MB, Ernstoff MS, Puzanov I, et al. Efficacy and safety of nivolumab (NIVO) plus ipilimumab (IPI) in patients with melanoma (MEL) metastatic to the brain: Results of the phase II study CheckMate 204. (Presented at ASCO 2017). Journal of Clinical Oncology. 2017; 35:9507-9507.

10. Postow MA, Chesney J, Pavlick AC, Robert C, Grossmann K, McDermott D, Linette GP, Meyer N, Giguere JK, Agarwala SS, Shaheen M, Ernstoff MS, Minor D, et al. Nivolumab and Ipilimumab versus Ipilimumab in Untreated Melanoma. N Engl J Med. 2015; 372:2006-17. https://doi. org/10.1056/NEJMoa1414428.

11. Wolchok JD, Chiarion-Sileni V, Gonzalez R, Rutkowski P, Grob JJ, Cowey CL, Lao CD, Wagstaff J, Schadendorf D, Ferrucci PF, Smylie M, Dummer R, Hill A, et al. Overall Survival with Combined Nivolumab and Ipilimumab in Advanced Melanoma. N Engl J Med. 2017; 377:1345-56. https://doi.org/10.1056/NEJMoa1709684.

12. Hammers HJ, Plimack E, Infante JR, Rini B, McDermott D, Ernstoff M, Voss M, Sharma P, Pal S, Razak A, Kollmannsberger C, Heng D, Spratlin J, et al. Expanded cohort results from CheckMate 016a: a phase I study of nivolumab in combination with ipilimumab in metastatic renal cell carcinoma (mRCC). Journal of Clinical Oncology. $2015 ; 33: 4516-4516$.

13. Long GV, Atkinson V, Cebon JS, Jameson MB, Fitzharris BM, McNeil CM, Hill AG, Ribas A, Atkins MB, Thompson JA, Hwu WJ, Hodi FS, Menzies AM, et al. Standarddose pembrolizumab in combination with reduceddose ipilimumab for patients with advanced melanoma (KEYNOTE-029): an open-label, phase 1b trial. Lancet Oncol. 2017; 18:1202-10. https://doi.org/10.1016/ S1470-2045(17)30428-X.

14. Weber JS, Gibney G, Sullivan RJ, Sosman JA, Slingluff CL, Lawrence DP, Logan TF, Schuchter LM, Nair S, Fecher L, Buchbinder EI, Berghorn E, Ruisi M, et al. Sequential administration of nivolumab and ipilimumab with a planned switch in patients with advanced melanoma (CheckMate 064): an open-label, randomised, phase 2 trial. Lancet Oncol. 2016; 17:943-55. https://doi.org/10.1016/ S1470-2045(16)30126-7.

15. Meerveld-Eggink A, Rozeman EA, Lalezari F, van Thienen JV, Haanen JBA, Blank CU. Short-term CTLA-4 blockade directly followed by PD-1 blockade in advanced melanoma patients: a single-center experience. Ann Oncol. 2017; 28:862-7. https://doi.org/10.1093/annonc/mdw692.

16. Postow M. Reduced-dose ipilimumab with standard-dose pembrolizumab: is less more? Lancet Oncol. 2017; 18:11445. https://doi.org/10.1016/S1470-2045(17)30518-1.

17. Kirchberger MC, Hauschild A, Schuler G, Heinzerling L. Combined low-dose ipilimumab and pembrolizumab after sequential ipilimumab and pembrolizumab failure in advanced melanoma. Eur J Cancer. 2016; 65:182-4. https:// doi.org/10.1016/j.ejca.2016.07.003.

18. Hellmann MD, Rizvi NA, Goldman JW, Gettinger SN, Borghaei H, Brahmer JR, Ready NE, Gerber DE, Chow LQ, Juergens RA, Shepherd FA, Laurie SA, Geese WJ, et al. Nivolumab plus ipilimumab as first-line treatment for advanced non-small-cell lung cancer (CheckMate 012): results of an open-label, phase 1, multicohort study. Lancet Oncol. 2017; 18:31-41. https://doi.org/10.1016/ S1470-2045(16)30624-6.

19. Costa R, Carneiro BA, Agulnik M, Rademaker AW, Pai SG, Villaflor VM, Cristofanilli M, Sosman JA, Giles FJ. Toxicity profile of approved anti-PD-1 monoclonal antibodies in solid tumors: a systematic review and metaanalysis of randomized clinical trials. Oncotarget. 2017; 8:8910-20. https://doi.org/10.18632/oncotarget.13315. 